\title{
An Approach for Recommendations in Self Management of Diabetes based on Expert System
}

\author{
Baran Hashemi \\ MSc in Nutrition
}

\author{
Hossein Javidnia \\ BEng in Information Technology Engineering
}

\begin{abstract}
Diabetes self-management education is of significant importance, since people with diabetes and their families provide $95 \%$ of their care themselves. Hyperglycemia and hypoglycemia are two of the most serious acute complications of diabetes. Making appropriate decision in these situations needs knowledge about normal blood glucose levels and related signs and symptoms. In this paper an expert system is proposed using Visual C\# 2008, aiming at nutrition recommendations applicable in blood glucose self management. This expert system consists of 4 sections: Body weight and daily nutritional requirements assessment, Hypo- and hyperglycemia symptoms, Self-monitoring of Blood Glucose (SMBG) and Diabetes related disease. In comparison with the other expert systems invented to manage blood glucose level, this expert system includes different aspects of diabetes and is usable for both experts and diabetes patients.
\end{abstract}

\section{Keywords}

Diabetes recommendation, Expert system, Hyperglycemia, Hypoglycemia

\section{INTRODUCTION}

Diabetes is globally increasing at an alarming rate. It is a complex, chronic condition that affects all areas of a person's life and requires high quality care [1]. According to International Diabetes Federation (IDF) 366 million people had diabetes in 2011 and by 2030 this will have risen to 552 millions [1-2]. Diabetes self-management education of blood glucose level is of significant importance, since people with diabetes and their families provide $95 \%$ of their care themselves [1]. Without appropriate education people cannot make complex daily medical decisions required for preserving good health quality and even survival [3]. Hyperglycemia and hypoglycemia are two of the most serious acute complications of diabetes [4-5]. Making appropriate decision in these situations needs knowledge about normal blood glucose levels and related signs and symptoms. An increasing number of software systems including web-based systems, knowledge-based expert systems and fuzzy expert systems are designed aiming at diabetes diagnosis and treatment [6-17]. Most of them can be employed in health care settings only [7-12]. In a study by Kalpana carried out in 2011 a fuzzy expert system based on fuzzy verdict mechanism was designed to justify physician's decision making in diabetes diagnosis. At first experimental data were processed and the values were converted into fuzzy values (fuzzification stage).Then the fuzzy verdict mechanism executed rules to make a decision on the possibility of presence of diabetes [15]. Just a few expert systems have been designed incorporating nutrition in self management of diabetes [6, 16-18].Campos-Delgado et al. in 2006 developed a Mamdani-type fuzzy-based system to present a blood glucose controller for type-1 diabetes patients under multiple daily insulin injections regimen [16].A diabetes self-management expert system, designed in 1992, resulted in significant improvement in knowledge and dietary habits of diabetes patients. It also showed significant reduction in HbA1c levels [17]. Jha developed a knowledge-based expert System which integrated natural treatment methods in diabetes management. The methods consisted of massage, acupuncture, herbal/proper nutrition and gems [6]. In another expert system designed by Kovasznai in 2011 for diet recommendation, nutrition recommendations for diabetes patients were also included [18].This article is organized as follows: In the first part of this paper expert system and the multi-agent system will be described, respectively. The second part is dedicated to author's proposed method.

\section{EXPERT SYSTEM}

In artificial intelligence, an expert system is a computer system that imitates the decision-making ability of an expert [19]. An expert system is a software system created in a way as to be able to use the ability of the user in dealing with problem successfully. It is divided into two parts, one fixed, independent of the expert system: the inference engine, and one variable: the knowledge base. The system uses knowledge and information of an expert and a set of rules which defined by a knowledge engineer to begins a process for the purpose of inferring a problem. The problem could probably be information or data collected in a study. In this paper, a doctor or a person capable of managing diabetes is considered to be the expert. The inputs of the expert system are the values seen in Figure 2. The expert system consists of the following four parts (Figure 1):

$\begin{array}{ll}\text { 1. } & \text { Agent } \\ \text { 2. } & \text { User Interface } \\ \text { 3. } & \text { Knowledge Base } \\ \text { 4. } & \text { Decision Making }\end{array}$

The sequence of operations carried out in this expert system can be seen in Figure 1.

As can be seen in Figure 1, a knowledge engineer interviews and meets experts and learns what they know and how they judge based on their knowledge. The engineer then translates the knowledge into a computer-usable language and designs an inference engine, a reasoning structure, which uses the 
knowledge appropriately. He also determines how to assimilate the use of uncertain knowledge in the reasoning process, and what kinds of details would be useful to the end user. Next, the inference engine and facilities for representing knowledge are programmed, and the domain knowledge is entered into the program piece by piece. It may be that the inference engine is not just right; the form of knowledge representation is uncooperative for the kind of knowledge needed for the task; and the expert might decide the pieces of knowledge are wrong. All these are discovered and modified as the expert system gradually gains competence.

\section{MULTI-AGENT SYSTEM}

A multi-agent system consists of several agents interacting reciprocally with other agents both within themselves and with their surrounding environment. An agent is either a digital computer program or a program capable of independent action. In other words, the agents are autonomous. Agents are sophisticated computer programs that act autonomously in support of their users, across open and distributed environments, to resolve complex problems. However, applications require multiple agents that can work together. A multi-agent system (MAS) is a coupled network of software agents that interact to resolve problems that are beyond the individual capacities or knowledge of each expert [20].The goal of multi-agent systems' research is to find methods that allow building complex systems composed of independent agents who, while operating on restricted knowledge and owning only limited abilities, are nevertheless capable of performing the desired global behaviors. In this paper, the multi- agent system has several agents such as fasting and pre-prandial blood glucose, postprandial ( 2 hours) blood glucose, bedtime blood glucose, random blood glucose, etc. In the following expert system (Figure 2), blood glucose test results in different situations are input parameters. According to the defined rules of the system, it is decided whether hypo- or hyperglycemia acquired or not. For each case associated recommendations will be appeared.

\section{PROPOSED METHOD}

The proposed expert system includes 4 internal separate sub-expert systems.

Section 1: Body weight and daily energy and macronutrient requirements assessment. This section contains two parts. One part determines daily requirements of calorie, carbohydrates, proteins and fats based on patient's weight, height, age, gender and physical activity level. Proteinuria is a condition of presence of protein in the urine and is the predictor of diabetes nephropathy. People with proteinuria have to consume less protein. In this case, system calculates protein lower than that of This paper proposed an expert system for nutrition recommendations in diabetes and related diseases. This expert system is feasible in diabetes self management and helps diabetes patients to make appropriate health related decisions in complex situations. Some expert systems were designed for diagnosis and treatment of diabetes [6, 16-18]. These expert systems did not include both type of diabetes (type 1 and 2) [16], diabetes complications (ex: proteinuria) [6, 16-18] blood glucose level in different situations (ex: pre exercise blood the same condition according to engineer-defined rules. Another part of this section calculates BMI (body mass index) and body fat percent and compares them with defined ranges [21-22]. In the case of overweight or obesity system declares what the normal ranges of weight and body fat percent are. After that, patient can get some weight reduction advices according to the sample code in Figure 3 and correspondent recommended message will be visualized to the user (Figure 4).

Section 2: Hypoglycemia and hyperglycemia symptoms. When hypo- or hyperglycemia develops different symptoms may occur. In this section if the patient mark the symptoms he/she experiences, the system is able to guess to which condition (ex: hyperglycemia) the symptoms may be related. It declares the patient his/her status and asks for blood glucose monitoring. Then the knowledge-based part and interface engine will provide the user with recommendations according to the level of the blood glucose (Figure 5). The recommendations are based on the type of diabetes (type 1 or type 2).

Section 3: Self-monitoring of Blood Glucose (SMBG). SMBG is an important component in effective and safe diabetes management. Patients may perform SMBG before meals, 1 to 2 hours after meals, at bedtime and during the night or whenever needed to know whether symptoms are caused by hypo- or hyperglycemia. Normal blood glucose levels have been classified for different ages according to the American Diabetes Association (ADbA) guidelines 2011 [23]. The included items are fasting/ pre-prandial, postprandial, bedtime, pre-exercise and random blood glucoses. In the case of hypoor hyperglycemia the system declares the patient that he/she may be at risk for hypo- or hyperglycemia and gives them appropriate recommendations. The recommendations are based on the types of diabetes (type 1 or type 2).

Let's consider someone who has low pre-prandial blood glucose $(<70 \mathrm{mg} / \mathrm{dl})$. The system announces that he/she is hypoglycemic. For his/her the first system recommendation is to consume 15 grams of a fast-acting carbohydrate and the second is to recheck his/her blood glucose fifteen minutes afterwards.

Section 4: Diabetes associated disease. Last section of this expert system contains a database of diseases which are associated with diabetes or may develop as a result of it such as overweight/ obesity, hypertension, hyperlipidemia, hypothyroidism, heart disease, celiac disease, gastroparesis, and diabetic neuropathy (Figure 7). If each of these problems is chosen, the related nutrition recommendations appear. Also definitions of key terms are available (Figure 8)

\section{CONCLUSION}

glucose) $[6,16-18]$ and diabetes associated diseases (ex: heart disease) $[6,16-18]$ in diabetes control. In this paper type of diabetes is information the system uses to conduct proper recommendations in hypo- or hyperglycemic conditions. Moreover, by using signs and symptoms of hypo- or hyperglycemia and patient's blood glucose level this system is able to make proper recommendations. By encompassing different aspects of diabetes and ease of use, this expert system is usable by both experts and diabetes patients. But there are 


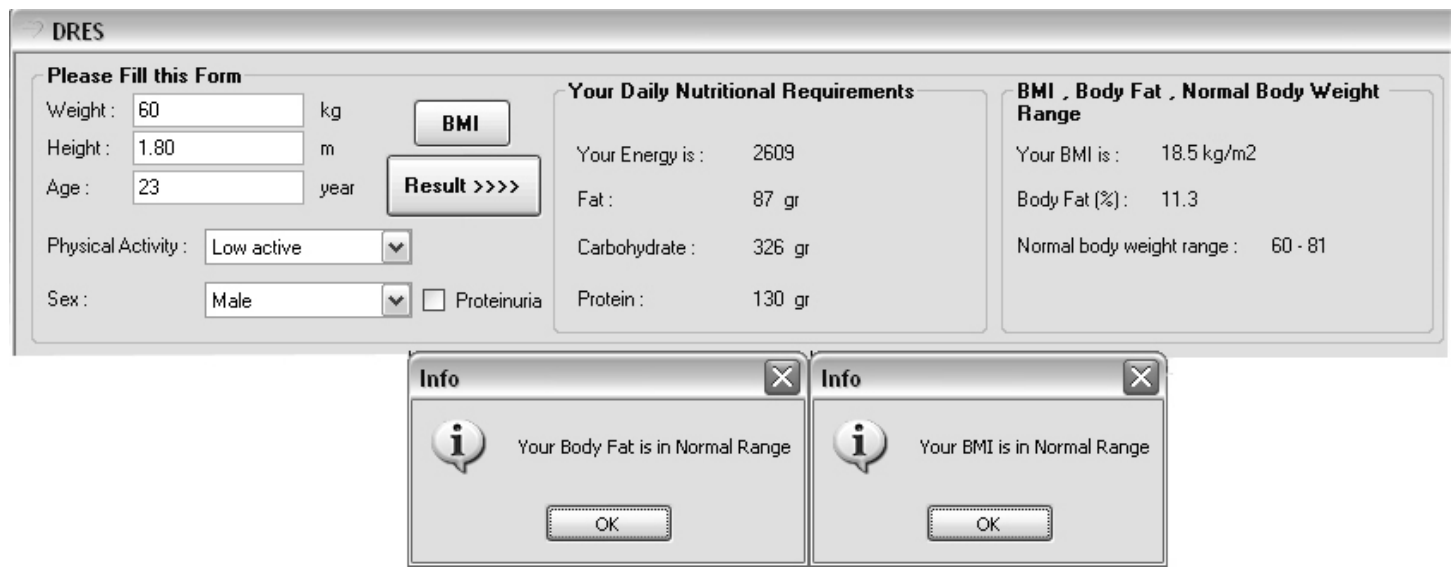

Figure 4: BMI and Body Fat Message

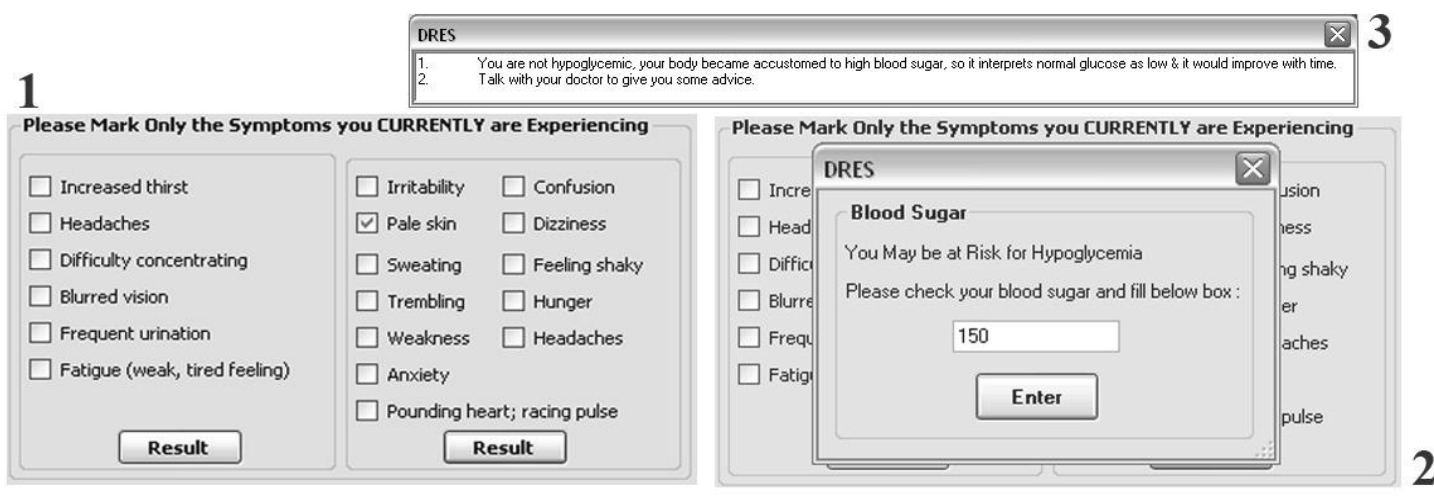

Figure 5: Symptoms of Hypo- and Hyperglycemia and related recommendation

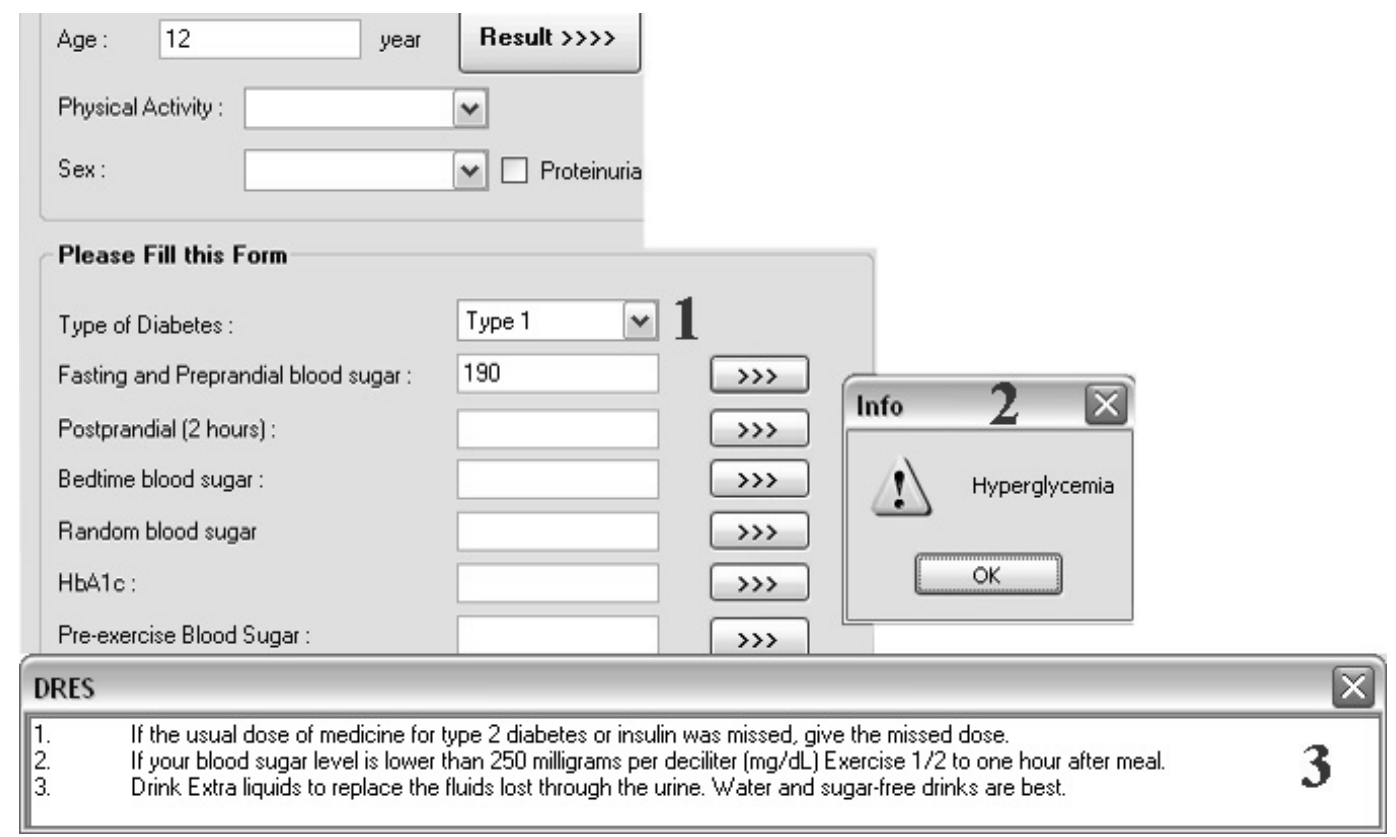

Figure 6: Sample Data and the Result in Phase 3 


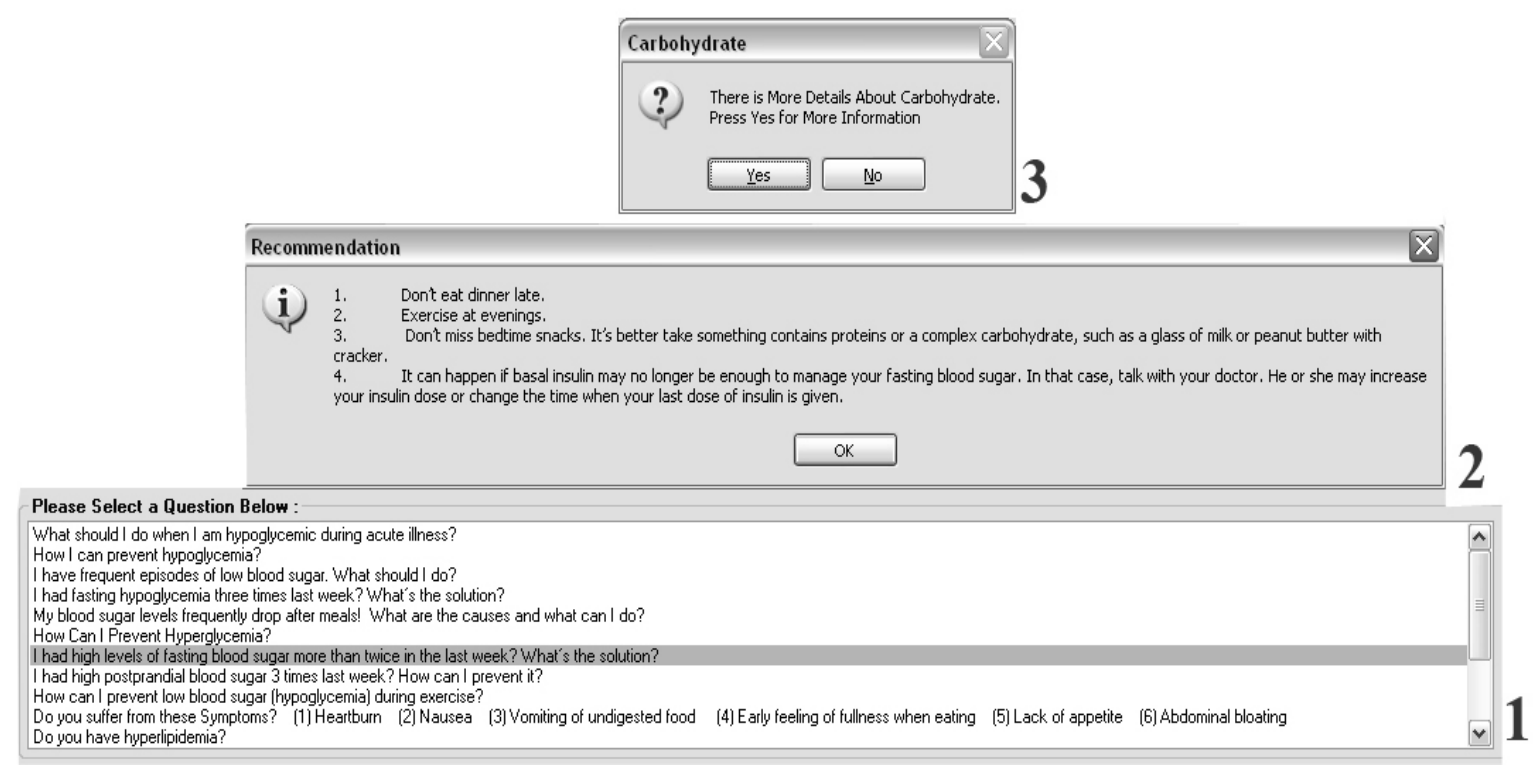

Figure 7: Last Part of Expert System

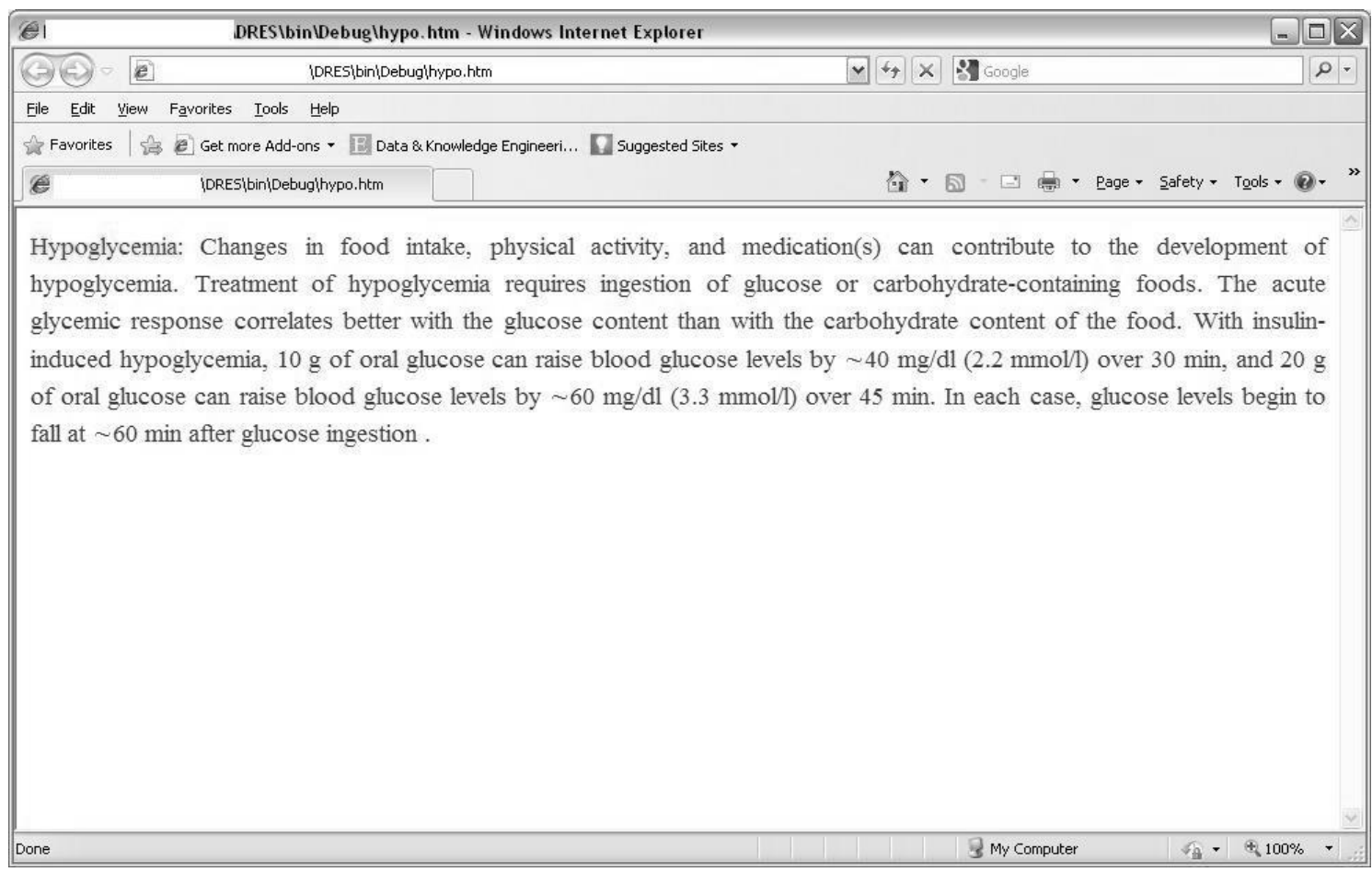

Figure 8: Sample definitions of key terms 


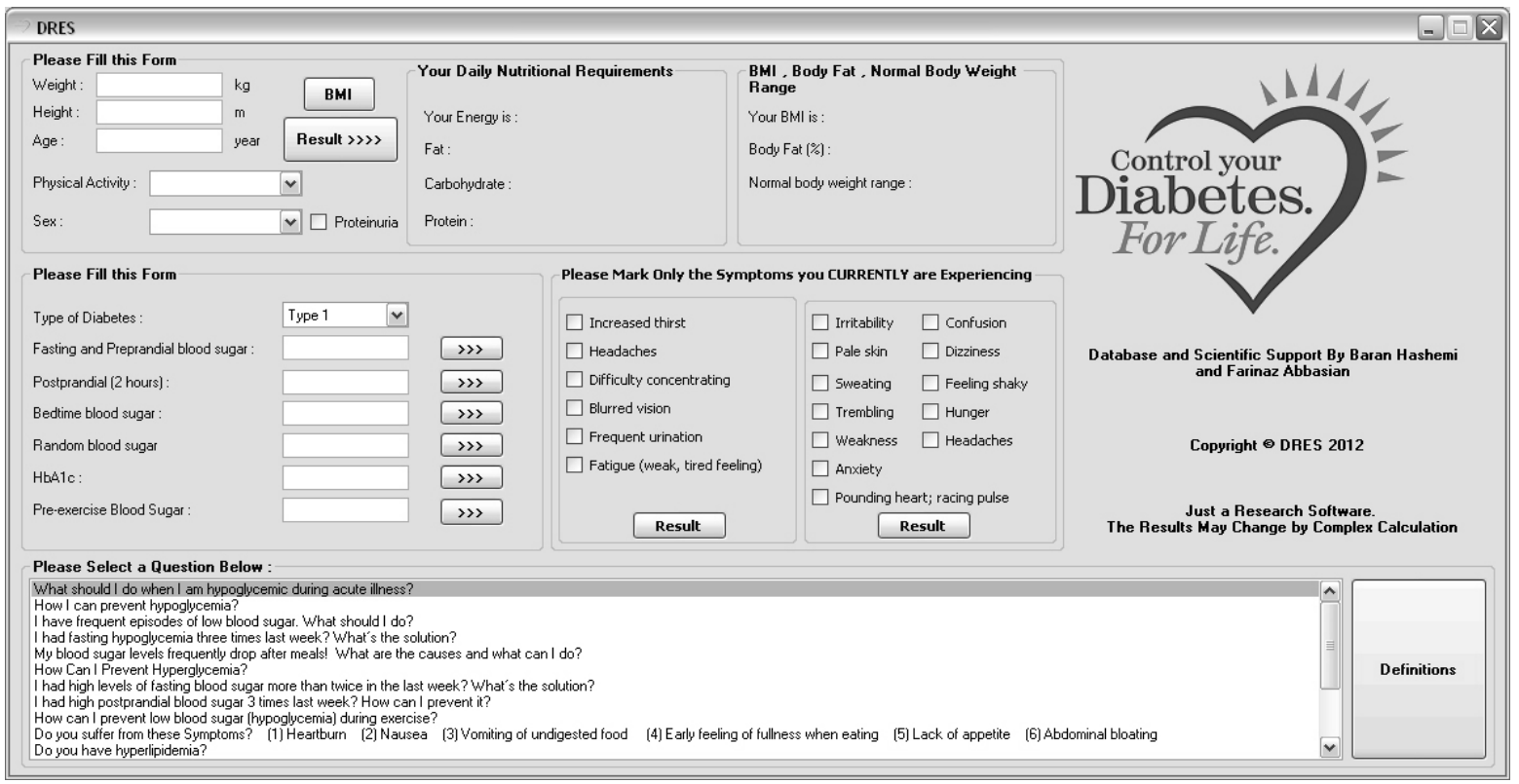

Figure 9: Proposed Program Whole Picture

\section{ACKNOWLEDGMENTS}

The authors thank Farinaz Abbasian for her participation in developing and testing the software.

\section{REFERENCES}

[1] International Diabetes Federation 2011. http://www.idf.org/diabetesatlas/5e/the-global-burden.

[2] World Health Organization 2011. http://www.who.int/mediacentre/factsheets/fs312/en/2011.

[3] Norris SL, Engelgau MM, Narayan KMV.2001 Effectiveness of self-management training in type 2 diabetes. A systematic review of randomized controlled trials. Diabetes Care 2001, 24:561-587.

[4] Perantie DC, Lim A, Wu J, Weaver P, Warren S L, Sadler M, White N H and Hershey T. 2008 Effects of prior hypoglycemia and hyperglycemia on cognition in children with type 1 diabetes mellitus. (Apr 2008), 9(2): 87-95.

[5] Goya A, Mehta S R, Díaz R, Gerstein H C, Afzal R, Xavier D, Liu L, Pais P and Yusuf S. 2009 Differential Clinical Outcomes Associated With Hypoglycemia and Hyperglycemia in Acute Myocardial Infarction (November 2009). doi: 10.1161/CIRCULATIONAHA.108.837765.

[6] Jha S K 2012 Development of knowledge base expert system for natural treatment of diabetes disease. IJACSA 3(3).

[7] Abu-naser s, El-Hissi H, Abu-Rass M, El-Khozondar N. 2010. An expert system for endocrine diagnosisand treatments using JESS. Journal of artificial intelligence 3(4): 239-251.

[8] Lee C. 2011.A Fuzzy Expert System for Diabetes Decision Support Application. IEEE digital library (Feb. 2011), 139153.

[9] Devi R E, Nagaveni N. 2010. Design methodology of a fuzzy knowledgebase system to predict the risk of diabetic nephropathy. International Journal of Computer Science Issues (September 2010), 7(5).

[10] Akter M, Uddin M S and Haque A. 2009. Diagnosis and management of diabetes mellitus through a knowledgebased system. 13th International Conference on Biomedical Engineering, 23(3) 1000-1003.

[11] Peters A N, Davidson M B. 1998. Application of a diabetes managed care program. Diabetes Care. (July 1998), 21(7)

[12] Rodbard D and Vigersky R A. 2011. Design of a decision support system to help clinicians manage glycemia in patients with type 2 diabetes mellitus. (March 2011). Journal of Diabetes Science and Technology, 5(2).

[13] Balas E A, Krishna S, Kretschmer R A, Cheek T R, Lobach D F and Boren S A. 2004. Computerized knowledge management in diabetes care. Medical Care (June 2004), 42(6).

[14] Yoon K -H, Kim H -S. 2007. A short message service by cellular phone in type 2 diabetic patients for 12 months. Elsevier Ireland Ltd.

[15] Kalpana M. 2011"Fuzzy Expert System for Diabetes using Fuzzy Verdict Mechanism", Int. J. Advanced Networking and Applications, 03(02):1128-1134

[16] Campos-Delgado D U, Hernandez-Ordonez M, Femat R and Gordillo-Moscos. 2006. Fuzzy-based controller for glucose regulation in type-1 diabetic patients by subcutaneous route, IEEE Trans. Biomed. Eng (Nov 2006), 53(11):2201-2210.

[17] Turnin M G, Beddok R H, Clottes J P, Martini P F, Abadie R G, Buisson J C 1992. Telematic expert system diabeto. Diabetes Care (Feb 1992)15(2).

[18] Kovasznai G 2011. Developing an expert system for diet recommendation. IEEE International Symposium on Applied Computational Intelligence and Informatics (May 2011), 19-21. 
International Journal of Computer Applications (0975 - 8887)

Volume 53-No.14, September 2012

[19] Peter J, Introduction to Expert Systems, Addison Wesley, p. 2, ISBN 978-0-201-87686-4. 1998 (3 ed.)

[20] School of Computer Science, Carnegie Mellon, Advanced Agent and Robotics Technology Lab.

[21] Centers for Disease Control (CDC) and Prevention: Overweight and obesity 2009, http://www.cdc.gov/obesity/.
[22] Deurenberg P, Deurenberg- Yap M. 2003. Validity of body composition methods across ethnic population groups. Acta Diabetol 40:246.

[23] American Diabetes Association (ADbA), 2011. Diagnosis and classification of diabetes mellitus (Position Statement), Diabetes Care 34(Suppl 1):S63, 2011 a. 